\title{
Differences in results of infrabony pocket treatment with addition of platelet rich fibrin and platelet rich plasma gel in DFDBA bone graft
}

\author{
Netta Anggraini ${ }^{*}{ }^{* *}$, Sri Pramestri Lastianny***, Al Sri Koes Soesilowati ${ }^{* * *}$ \\ *Departement of Periodontics, Faculty of Dentistry, Universitas Baiturrahmah, Padang, West Sumatra, Indonesia \\ **Periodontics Specialty Program, Faculty of Dentistry, Universitas Gadjah Mada, Yogyakarta, Indonesia \\ ***Departement of Periodontics, Faculty of Dentistry, Universitas Gadjah Mada, Yogyakarta, Indonesia \\ ***JI Denta No 1, Sekip Utara, Yogyakarta, Indonesia; e-mail: sri.pramestri@ugm.ac.id
}

Submitted: 24 ${ }^{\text {th }}$ July 2018; Revised: 29th August 2018; Accepted: $5^{\text {th }}$ December 2018

\begin{abstract}
Treatment of infrabony pocket makes use of bone graft material demineralized freeze dried bone allograft (DFDBA) from different individuals which has undergone demineralization process and it is osteoinductive. Enhancement of growth factor was done by adding platelet rich fibrin (PRF) and platelet rich plasma (PRP). PRP is activated with an addition of calcium chloride $\left(\mathrm{CaCl}_{2}\right)$ to form gel. The method used to apply the bone grafting material is open flap debridement (OFD). This research aimed to reveal the differences in the results of infrabony pocket treatment using PRF and PRP gel with an addition of DFDBA. The sample was taken from 20 infrabony pocket points divided into 2 groups, 10 infrabony pocket were treated with OFD+DFDBA+PRF and the other were treated with OFD+DFDBA+PRP gel. Pocket depth (PD) was measured on the baseline and the first and third month after treatment. Alveolar bone height was measured using cone beam computed tomography (CBCT) radiograph on the baseline to the third month after treatment. The results of this research showed that there was difference in the results of infrabony pocket treatment using PRF and PRP gel with an addition of DFDBA which could be observed from a reduction in PD from the baseline, month 1 and month 3 as well as reduction in alveolar bone height from the baseline to month 3 . This research concluded that infrabony pocket treatment PRF application yields better results than PRP gel application in terms of PD and alveolar bone height reduction.
\end{abstract}

Keywords: DFDBA; infrabony pocket; open flap debridement; PRF; PRP gel

\section{INTRODUCTION}

Periodontal pocket, also known as true pocket, is the main clinical symptom of periodontal diseases. Infrabony pocket is a pocket of which the base is more apical to the level of the adjacent alveolar bone, therefore the lateral pocket wall lies between the root surface and alveolar bone. ${ }^{1}$ The main purpose of periodontal disease treatment is for periodontal tissue regeneration as a result of infection, including alveolar bone, periodontal ligament and cementum. Treatment of periodontal disease protects and maintains the oral health of patients in order to continue to function optimally in terms of mastication, comfort and aesthetics. ${ }^{2} \mathrm{~A}$ number of treatments for periodontal diseases have been developed, the most often used technique is flap surgery. ${ }^{1}$
There are various materials used in periodontal treatment including guided tissue regeneration (GTR), graft material, application of growth factor, with an objective to stimulate periodontal tissue regeneration especially infrabony defect. PRF contains glycosaminoglycan (heparin, hyaluronic acid), transforming growth factor- $\beta 1$ (TGF $\beta-1$ ), platelet derived growth factors (PDGFs), insulinlike growth factor 3 (IGF), interleukin-1 $\beta$ (IL-1 $\beta$ ), interleukin 6 (IL-6), tumor necrosis factor $\alpha$ (TNF $\alpha$ ), interleukin 4 (IL-4), and vascular endothelial growth factor (VEGF). ${ }^{3}$

Platelet-rich plasma (PRP) is autologous platelet in plasma concentrated by centrifugation. PRP contains growth factor such as PDGF, TGF- $\beta$, and VEGF. ${ }^{4}$ PRP can be activated (usually by $\mathrm{CaCl}_{2}$ and/or thrombin) and applied over wound 
area. Growth factor will be released gradually when platelet gel is formed using $\mathrm{CaCl}_{2}$. $\mathrm{CaCl}_{2}$ activates and clots PRP by forming autogenous thrombin from prothrombin, resulting in the formation of a loose fibrin matrix which will release growth factor over 7 days. $^{5}$

Demineralized Freeze Dried Bone Allograft (DFDBA) is bone graft decalcified in hydrochloric acid then freeze dried, it can function as osteoinductor thus inducing new bone formation and stimulating mesenchymal cell maturation. ${ }^{6}$ Wound healing is a dynamic, continuous, overlapping process which consists of several phases, namely hemostasis, inflammation, differentiation, proliferation, migration of mesenchymal cells to wound area, angiogenesis, re-epithelialization, synthesis and regulation of collagen, vascular maturation or remodeling. ${ }^{7,8}$ Bone healing occurs one month after bone graft is performed, and after three months it becomes obvious on a radiographic examination. Maturation of bone graft in laminar bone requires varied healing time, ranging from 3 to 6 months, and depends on various factors such as age, wound healing factor, size of wound, bone damage in the grafting area. ${ }^{8}$ Based on the above background, we were interested in conducting a research to reveal differences in results of infrabony pocket treatment with addition of PRF and PRP gel in DFDBA bone graft at periodontal clinic Prof. Soedomo UGM Dental Hospital.

\section{MATERIALS AND METHODS}

This was a quasi-experimental research. The inclusion criteria consisted of patients aged 21-60 years old suffering from chronic periodontitis with pocket depth $>5 \mathrm{~mm}$ and with damage to infrabony bone. They were willing to sign an informed consent. The exclusion criteria were smokers/ alcoholic drinkers, taking long-term drugs, unwilling to undergo a surgical procedure, unwilling to join the research until finished.

The samples in this study consisted of 20 infrabony pocket points in patients with chronic periodontitis, divided into 2 treatment groups. Group Aconsisted of 10 infrabony pockets treated with OFD, DFDBA and PRF. Group B consisted of 10 infrabony pocket treated with Open Flap Debridement (OFD), DFDBA and PRP gel. Pocket depth (PD) was measured before the surgery (baseline), the first and third month after the surgery. Bone height was measured before the surgery (baseline) and in the 3rd month after the surgery.

This research was conducted in the following stages, application for ethical clearance to the dental research ethics commission Faculty of Dentistry UGM No 001379/KKEP/FKG-UGM/ $\mathrm{EC} / 2018$, clinical examination, measurement of plaque index and initial phase therapy consisting of dental health education (DHE) as well as scaling and root planning, occlusion adjustment if there was occlusal trauma and splinting if there were shaking teeth with a mobility greater than 1 degree. Measurement and recording of plaque index (PI), initial data collection, and measurement of PD and bone height were done in the teeth to be treated. Extraoral CBCT was done to measure bone height.

PRF was made by taking $10 \mathrm{ml}$ blood sample intravenously from patients using syringe, then transferred to a tube and centrifuged at 2700 revolutions per minutes ( $\mathrm{rpm}$ ) for 12 minutes. The centrifugation resulted in three layers, i.e. the top most layer contained acellular plasma, the middle layer contained PRF, and the bottom most layer contained red blood cells, then PRF was segregated from the second layer. ${ }^{9}$

Preparation of Platelet-rich plasma was prepared one hour before surgery, by taking $9 \mathrm{ml}$ blood from each of the subjects from their basilic vein to be put in a $10 \mathrm{ml}$ tube containing $1 \mathrm{ml}$ sodium citrate anticoagulant $3.8 \%$ and $9 \mathrm{ml}$ blood. The first centrifugation was done at $2400 \mathrm{rpm}$ for 10 minutes. The blood in the tube was separated into two layers, i.e. the bottom most layer containing red blood cells and the top most layer containing blood plasma and buffy coat or platelet pool. Aspiration of the top layer was done using a pipette then put in another tube, centrifuged at $3600 \mathrm{rpm}$ for 15 minutes. Afterwards, the top layer was aspirated as much as $2 / 3$ from the volume of the supernatant, while the remaining $1 / 3$ lower part contained platelets, a small amount of blood cells and plasma, named PRP. ${ }^{10}$ 
PRP gel was prepared immediately before application. At the first stage, autologous thrombin was made by taking $4 \mathrm{ml}$ autologous blood into a tube and left for \pm 30 minutes until it coagulated. The tube was then centrifuged at $3600 \mathrm{rpm}$ for 12 minutes. The top most layer of the supernatant was collected as autologous thrombin. PRP gel was made by mixing $0.1 \mathrm{ml}$ autologous thrombin with $0.03 \mathrm{ml} \mathrm{CaCl}_{2} 10 \%$. The ratio of PRP and autologous thrombin was 10:1.11

The anesthesia used for periodontal flap surgery was local anesthesia, injected after the surgical area was first disinfected. Vertical and sulcular full thickness flap incisions were performed. The flap was elevated and debridement was performed in bone defect by scaling root planning and curettage. Solution of $75 \mathrm{mg} / \mathrm{ml}$ tetracycline $\mathrm{HCl}$ was applied to the hard tissue for 3 minutes, and rinsed using distilled water. DFDBA bone graft material was applied, combined with PRF and PRP gel on the surgical area, then the flap was returned and sutured with an uninterrupted vertical mattress suture technique, then covered with periodontal dressing. The patients were given amoxycilin antibiotics with a dose of $500 \mathrm{mg}$ for 5 days, analgesics and antiinflamatory drugs. They were asked to return for PD clinical parameter measurement, 1 month and 3 months after the surgery. The second X-ray image was taken 3 months after the surgery. PD was measured using periodontal probe UNC 15. Alveolar bone height was measured using software on CBCT images in sagittal, longitudinal and axial sections.

The data from the results of this research were analyzed using SPSS IBM version 23.0. Normality test was performed to the results of pocket depth (PD) measurement according to the observation time within the same group using Shapiro-Wilk test, followed with non parametric test. The data of PD reduction among groups were analyzed using $U$ Mann-Whitney test.

Normality test was also performed to the analysis results of alveolar bone height using Shapiro-Wilk test, followed by parametric paired t-test. The data of bone height reduction among the groups was analyzed using independent t-test.

\section{RESULTS}

The data collected in this study were the results of clinical parameter measurement and radiological examination in the two groups of patients who suffered from chronic periodontitis with infrabony bone damage. The first group was treated with periodontal flap surgery with an addition of DFDBA and PRF, while the second group was treated with periodontal flap surgery with an addition of DFDBA and PRP gel.

Table 1 indicates that the highest mean of PD was obtained by the group with OFD+DFDBA+PRF on the baseline with a mean and standard deviation of $6.70 \pm 0.48 \mathrm{~mm}$. The lowest mean of PD was found in the group with OFD+DFDBA+PRP gel in the third month with a mean and standard deviation of $2.50 \pm 0.52 \mathrm{~mm}$.

The data in Table 2 show that there was PD reduction in both groups with the highest reduction in the group with OFD+DFDBA+PRF from the baseline to the third month. The highest mean of PD reduction was in the group with OFD+DFDBA+PRF from the baseline to month 3 , i.e. $4.20 \pm 0.79 \mathrm{~mm}$ and the lowest reduction of PD was in month 1 to month 3 in the second group, i.e. $1.20 \pm 0.79 \mathrm{~mm}$.

Normality test with Shapiro-Wilk test and homogeneity test with Levene test were performed on the data distribution in each of the groups. Table 3 below shows the results of the Shapiro-Wilk test on the data of PD reduction.

Table 1. Mean and standard deviation of pocket depth (PD) according to observation time and treatment group $(\mathrm{mm})$

\begin{tabular}{|c|c|c|c|}
\hline \multirow{2}{*}{ Time } & \multirow{2}{*}{$\mathrm{n}$} & \multicolumn{2}{|c|}{ OFD + DFDBA } \\
\hline & & PRF & PRP Gel \\
\hline Baseline & 10 & $6.70 \pm 0.48$ & $6.40 \pm 0.51$ \\
\hline Month 1 & 10 & $3.70 \pm 0.67$ & $4.20 \pm 0.67$ \\
\hline Month 3 & 10 & $2.50 \pm 0.52$ & $3.00 \pm 0.81$ \\
\hline
\end{tabular}

Table 2. Mean and standard deviation of PD reduction between treatment groups $(\mathrm{mm})$

\begin{tabular}{lccc}
\hline \multirow{2}{*}{ Time } & \multirow{2}{*}{$\mathrm{n}$} & \multicolumn{2}{c}{ OFD + DFDBA } \\
\cline { 3 - 4 } & & PRF & PRP Gel \\
\hline Baseline - month 1 & 10 & $3.00 \pm 0.81$ & $2.20 \pm 0.63$ \\
Baseline - month 3 & 10 & $4.20 \pm 0.79$ & $3.40 \pm 0.84$ \\
Month 1- month 3 & 10 & $1.20 \pm 0.79$ & $3.00 \pm 0.79$ \\
\hline
\end{tabular}


Majalah Kedokteran Gigi Indonesia. April 2019; 5(1): 29 - 34

ISSN 2460-0164 (print)

ISSN 2442-2576 (online)

Table 3. Results of Shapiro-Wilk test on PD reduction

\begin{tabular}{ccc}
\hline OFD+DFDBA & Significance level & Description \\
\hline PRF month 0-1 & 0.035 & Abnormal \\
PRF month 0-3 & 0.023 & Abnormal \\
PRF month 1-3 & 0.004 & Abnormal \\
PRP gel month 0-1 & 0.012 & Abnormal \\
PRP gel month 0-3 & 0.172 & Normal \\
PRP gel month 1-3 & 0.004 & Abnormal \\
\hline
\end{tabular}

Table 4. Results of Mann Whitney test on PD reduction among observation time and treatment groups

\begin{tabular}{ccc}
\hline Mann whitney & Significance level & Description \\
\hline Baseline - month 1 & 0.035 & Significant \\
Baseline - month 3 & 0.046 & Significant \\
Month 1 - month 3 & 1.00 & Not significant \\
\hline
\end{tabular}

Table 5. Mean and standard deviation of alveolar bone height according to observation time and treatment groups

\begin{tabular}{|c|c|c|c|}
\hline \multirow{2}{*}{ Time } & \multirow{2}{*}{$\mathrm{n}$} & \multicolumn{2}{|c|}{ OFD+ DFDBA } \\
\hline & & PRF & PRP gel \\
\hline Baseline & 10 & $6.01 \pm 1.57$ & $6.11 \pm 0.88$ \\
\hline Month 3 & 10 & $3.96 \pm 0.92$ & $4.92 \pm 0.63$ \\
\hline
\end{tabular}

This means that the data distribution in both groups was abnormal. Since the Shapiro-Wilk test resulted in abnormal data distribution, the statistical test was continued with non parametric Mann Whitney test

Table 4 shows the results of Mann Whitney test on PD reduction with a significance level of $p<0.05$, indicating there was statistically significant difference between the groups when measured from the baseline to month 1 , baseline to month 3 , while the significance level of the measurement from month 1 to month 3 was $p>0.05$, meaning that there was no statistically significant difference between these groups.

Table 5 shows the that highest mean of bone height was found in the group of OFD+DFDBA+PRP gel in month 0 (baseline), is $6.11 \pm 0.88 \mathrm{~mm}$, while the lowest one was in the group of OFD+DFDBA+PRF in month 3 , is $3.96 \pm 0.92 \mathrm{~mm}$. Table 6 shows that the highest bone height reduction was found in the group of OFD+DFDBA+PRF from the baseline to month 3, i.e. $2.05 \pm 0.93 \mathrm{~mm}$ while the lowest reduction was in the group of OFD+DFDBA+PRP gel from the baseline to month 3, i.e. $1.19 \pm 0.82$ $\mathrm{mm}$.

The data distribution in each of the groups was tested using the Shapiro-Wilk normality test and Levene homogeneity test. Table 7 below presents the results of the Shapiro-Wilk normality test on the data of bone height reduction.

The Shapiro-Wilk normality test, as presented in Table 7, resulted in a significance level $p>0.05$ in the groups of OFD+DFDBA+PRF and OFD+DFDBA+PRP gel in month 0 . This indicates that these groups had normal data distribution.

The homogeneity test in Table 8 resulted in a significance level of $p>0.05$ in the groups of OFD+DFDBA+PRF and OFD+DFDBA+PRP gel, so the data were homogeneous. These tests showed that the groups had normal data distribution and the data were homogeneous, thus followed by a statistical test in the form of parametric Independent t-test. Table 9 shows that coronal bone height reduction from the baseline to month 3 had $p<0.05$, meaning that there was a statistically significant difference in the reduction of bone height in the baseline and in month 3 between the two treatment groups.

Table 6. Mean and standard deviation of alveolar bone height reduction according to treatment groups

\begin{tabular}{|c|c|c|c|}
\hline \multirow{2}{*}{ Time } & \multirow{2}{*}{$\mathrm{n}$} & \multicolumn{2}{|c|}{ OFD+ DFDBA } \\
\hline & & PRF & PRP gel \\
\hline Baseline- month 3 & 10 & $2.05 \pm 0.93$ & $1.19 \pm 0.82$ \\
\hline
\end{tabular}

Table 7. Results of shapiro-wilk test on alveolar bone height reduction

\begin{tabular}{ccc}
\hline $\begin{array}{c}\text { Group } \\
\text { OFD+DFDBA }\end{array}$ & $\begin{array}{c}\text { Significance } \\
\text { level }(p)\end{array}$ & Description \\
\hline PRF month 0 - month 3 & 0.223 & Normal \\
PRP gel month 0-3 & 0.177 & Normal \\
\hline
\end{tabular}

Table 8. Results of homogenity test on alveolar bone height reduction

\begin{tabular}{ccc}
\hline Levene statistic & Significance level $(p)$ & Description \\
\hline & 0.669 & Homogeneous \\
\hline
\end{tabular}

Table 9. Results of independent t-test on alveolar bone height reduction among treatment groups

\begin{tabular}{lcl}
\hline & Significance level & Description \\
\hline Baseline- month 3 & 0.042 & Significant \\
\hline
\end{tabular}




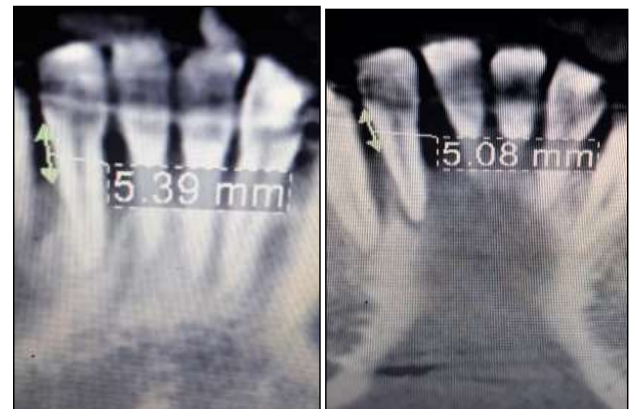

(A)

(B)

Figure 1. Images of alveolar bone height $(A)$ baseline (B) month 3 treatment with PRF

\section{DISCUSSION}

PD reduction from the baseline to month 1 and from the baseline to month 3 showed a significant difference between the two treatment groups. The reduction in the group of OFD+DFDBA+PRF from the baseline to month 3 was greater than that of OFD+DFDBA+ PRP gel indicating that the group of OFD+DFDBA+PRF underwent angiogenesis, allowing regeneration process to take place optimally. the Growth factor contained in PRF is capable of increasing the formation of cementum and proliferation of periodontal ligament fibroblasts. ${ }^{9}$ Receptors of growth factor are found in periodontal ligament which will activate fibroblasts. PRP gel produces less IGF-1 compared to PRF. The growth factors contained in PRP play a role as a biological mediator which affects proliferation, differentiation, migration and attachment of periodontal progenitor cells. ${ }^{11}$ Unfortunately the process of making PRP requires additional anticoagulants in large quantities and also bovine-derived thrombin which functions to convert fibrinogen to fibrin. The addition of thrombin from bovine makes PRP not purely autologous so that side effects are obtained in the form of antibodies to factor $\mathrm{V}, \mathrm{XI}$ and thrombin which results in coagulopathy. ${ }^{12}$

The results of reduction from month 1 to month 3 showed no significant difference between the two treatment groups, meaning that in general the two treatment groups underwent the same regeneration process. Wound healing immediately took place over time after the treatment was given and started from hemostasis, inflammatory, proliferative and remodeling phases..$^{13}$ In hemostasis phase, space between flap and bone surface is filled with blood clots consisting of fibrin threads with large amounts of polymorphonuclear leukocytes, erythrocytes, and debris from damaged cells as well as capillaries at the edges of the wound. After the hemostasis phase, wound healing enters the inflammatory phase which reaches its peak on day 2-3, proliferative phase which reaches its peak in week 2-3, then remodeling phase which starts during the peak of proliferative phase, i.e. week 3 or day 21 , and the bone regeneration process takes place $\pm 1-3$ months; one of the success parameters of periodontitis treatment can be seen from PD reduction. ${ }^{1,14}$

The data showed that the highest mean of alveolar bone height reduction was found in the group of OFD+DFDBA+PRF from the baseline to month 3 , while the lowest mean of bone height reduction was in the group of OFD+DFDBA+PRP gel from the baseline to month 3 . A high alveolar bone height reduction results in lower alveolar bone height, indicating bone repair. Increased alveolar bone repair shows periodontal tissue regeneration which can be seen in X-ray images in the form of a radiopaque area in alveolar bone. ${ }^{15}$

Difference in bone height in the group of $O D F+D F D B A+P R F$ and that of OFD+DFDBA+PRP gel shows a significant result, meaning that there was a significant difference between the two groups from the baseline to month 3 . Activation of platelet rich plasma in this study used $\mathrm{CaCl}_{2}$ which activated the growth factors contained in PRP. $\mathrm{CaCl}_{2}$ added after the second centrifugation resulted in a more gel-like PRP consistency, making it easily applied to bone defect or damage. Addition of $\mathrm{CaCl}_{2}$ could stimulate the release of platelet growth factor and increase the proliferation and migration of osteoblasts. The use of PRF will be more effective because PRF clots form a strong 3-dimensional fibrin matrix, and unlike PRP gel, fibrin matrix will be re-formed gradually with a pattern similar to that of natural blood clot matrix formation. ${ }^{16}$

\section{CONCLUSION}

This study concluded that there are differences in the results of infrabony pocket treatment using 
platelet rich fibrin and platelet rich plasma gel with an addition of demineralized freeze dried bone allograft. This is evident from the fact that pocket depth reduction and alveolar bone height repair due to the application of PRF are greater than that of PRP gel. Based on the results of this study, it is recommended that a research with a longer observation time be conducted in order to see the stability of alveolar bone. In addition, it is necessary to perform patients' blood tests prior to taking blood sample for the making of PRF and PRP gel.

\section{REFERENCES}

1. Newman MG, Takei HH, Carranza FA, Klokkevold PR. Carranza's Clinical Periodontology $11^{\text {th }}$ ed. Philadelphia: W.B. Saunders Co; 2012. 550-555, 587.

2. Xiang-Ying $O$, Jing $Q$. Effect of plateletrich plasma in the treatment of periodontal intrabony defects in humans. Chin Med J (Engl). 2006; 119(18): 1511-1521.

3. Kumar RV, Shubhashini N. Platelet rich fibrin: a new paradigm in periodontal regeneration. Cell Tissue Bank. 2012; 14(3): 453-463. doi: 10.1007/s10561-012-9349-6

4. Ouyang $X Y$, Qiao J. Effect of platelet-rich plasma in the treatment of periodontal infrabony defects in humans. Chinese Medical Journal. 2006; 119(18): 1511-1521. doi: 10.1097/00029330-200609020-00003

5. Rodriguez IA, Kalaf EA, Bowlin GL, Sell SA. Platelet-Rich plasma in bone regeneration: engineering the delivery for improved clinical efficacy. Biomed Res Int. 2014; 2014: 392398. doi: 10.1155/2014/392398

6. Bender SA, Rogalski JB, Mills MP, Arnold RM, Cochran DL, Mellonig JT. Evaluation of demineralized bone matrix paste and putty in periodontal intraosseous defects. J Periodontol. 2005; 76(5): 768-777. doi: 10.1902/jop.2005.76.5.768

7. Guo S, DiPietro LA. Factors affecting wound healing. J Dent Res. 2010; 89(3): 219-229. doi: $10.1177 / 0022034509359125$
8. Giele H, Cassell O. Plastic and reconstructive surgery. Oxford Specialist Handbook in Surgery; 2008.

9. Dohan DM, Choukroun J, Diss A, Dohan SL, Dohan AJ, Mouhyi J, Gogly B. Platelet-Rich Fibrin (PRF): a second-generation platelet concentrate. Part I: technological concepts and evolution. Oral Surg Oral Med Oral Pathol Oral Radiol Endod. 2006; 101(3): e37-44. doi: 10.1016/j.tripleo.2005.07.008

10. Lee KS, Wilson JJ, Rabago DP, Baer GS, Jacobson JA, Borrero GC. Musculosceletal applications of platelet-rich plasma: fad or future? AJR Am J Roentgenol. 2011; 196(3): 628-636. doi: 10.2214/AJR.10.5975

11. So SG, Lee JY, Park JB, Kim HC, Park IH, Lee SC. Using autologous thrombin in making prp gel -case reports-. Living Well Institute of Dental Research. 2011; 60-69.

12. Raja V Sunitha, Naidu E Murinatham. 2008. Platelet-rich Fibrin: Evolution of a secondgeneration platelet concentrate. Indian Journal of Dental Research, 2008; 19(1):42-46

13. Javed F, Askar MA, Rasheed AA, Hezaimi KA. Significance of the paltelet-derived growth factor in periodontal tissue regeneration. Arch Oral Biol. 2011; 56(12): 1476-1484. doi: 10.1016/j.archoralbio.2011.06.020

14. Lindhe J, Lang NP, Karring T. Clinical Periodontology and Implant Dentistry. $5^{\text {th }}$ ed. United Kingdom: Blackwell Munksgard; 2008. 57-59.

15. Yilmaz S, Cakar G, Ipci SD. Platelet rich plasma in reconstructive periodontal therapy. Platelets. 2011; 23: 269-290. doi: $10.5772 / 22967$

16. Toit JD, Gluckman H, Salama M. Platelet-Rich Fibrin (PRF): a growth factor-rich biomaterial. part 1 - the platelet concentrates milieu \& review of the literature. International Dentistry - African Edition. 2015; 5(5): 62-70. 